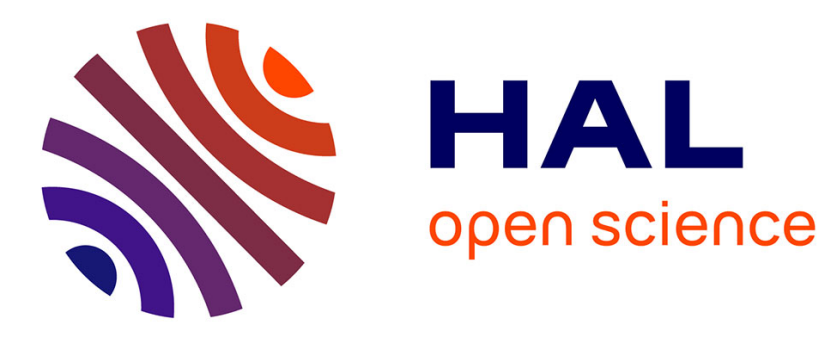

\title{
Thick multi-layers analysis using high energy PIXE
}

Alexandre Subercaze, Arnaud Guertin, Ferid Haddad, Charbel Koumeir, Vincent Métivier, Noël Servagent

\section{To cite this version:}

Alexandre Subercaze, Arnaud Guertin, Ferid Haddad, Charbel Koumeir, Vincent Métivier, et al.. Thick multi-layers analysis using high energy PIXE. Nuclear Instruments and Methods in Physics Research Section B: Beam Interactions with Materials and Atoms, 2017, 406, pp.104-107. 10.1016/j.nimb.2017.02.014 . hal-02912587

\section{HAL Id: hal-02912587 https://hal.science/hal-02912587}

Submitted on 29 Jan 2021

HAL is a multi-disciplinary open access archive for the deposit and dissemination of scientific research documents, whether they are published or not. The documents may come from teaching and research institutions in France or abroad, or from public or private research centers.
L'archive ouverte pluridisciplinaire HAL, est destinée au dépôt et à la diffusion de documents scientifiques de niveau recherche, publiés ou non, émanant des établissements d'enseignement et de recherche français ou étrangers, des laboratoires publics ou privés. 


\title{
Thick multi-layers analysis using high energy PIXE
}

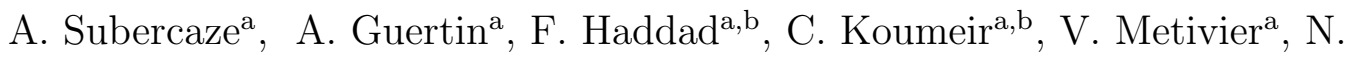 \\ Servagent $^{\mathrm{a}}$ \\ ${ }^{a}$ Laboratoire SUBATECH, Ecole des mines de Nantes, Université de Nantes, \\ CNRS/IN2P3, 4 Rue Alfred Kastler, 44307 Nantes cedex 3 - FRANCE \\ ${ }^{b}$ GIP ARRONAX, 1 rue ARRONAX, 44817 Saint-Herblain cedex - FRANCE
}

\begin{abstract}
A method for multi-layer analysis using high energy PIXE is described. It is based on the variation of the $\frac{K_{\alpha}}{K_{\beta}}$ ratio as a function of the detection angle. This method can provides the thicknesses and the sequences of multi-layers targets. Experiments have been carried out at the ARRONAX cyclotron using $70 \mathrm{MeV}$ protons in order to test this method. The thicknesses and the sequences of simple multi-layers targets and also of more complex targets with hidden layers have been determined using this method.
\end{abstract}

Keywords: Ion beam analysis; High energy PIXE; Multi-layers.

\section{Introduction}

High energy PIXE (HEPIXE), a technique using protons with energies higher than $30 \mathrm{MeV}$, has already been used to investigate piece of art and especially paintings [1-3]. High energy PIXE is a well-suited method for thick multi-layer analysis due to the large range of high energy protons. Using high energy particles beam allows to perform analysis in air and thus reduce the constraint on the samples preparation. The low energy loss of the high energy beam combined with a smooth variation of the $\mathrm{K}$ X-ray production cross sections in this energy range induced a low variation of this cross section during the experiments. The data analysis is therefore simplified. So far, HEPIXE has been able to give the thicknesses and the sequences of the layers [4] using $\frac{K_{\alpha}}{K_{\beta}}$ (or $\frac{K_{\alpha}}{L_{\alpha}}$ ) ratio, but hypothesis on the sample composition is required. We have already developed a method [5] to determine the thicknesses and the sequences of a simple ideal case. Those ideal targets were composed of layers of pure material foils with no repetition 
of an element and without hidden layers. However, in real applications of multi-layer analysis (such as paintings or coins) there might be some layers composed of light elements, located at the surface or in the bulk that cannot be detected (hidden layers). Therefore, the method presented in [5] cannot be applied. In this work, we present a method to determine the thicknesses and the sequences of multi-layer targets with hidden layers.

\section{Analysis method}

We need some observables sensitive to the thickness and the sequence of layers. For that purpose, we study the influence of the detection angle, the

angle between the sample and the detector, on the $\frac{K_{\alpha}}{K_{\beta}}$ ratio. The number of the detected $K_{\alpha} \mathrm{X}$-rays is given by the equation :

$$
N_{K_{\alpha}}=N_{p} \cdot \epsilon_{\alpha} \cdot \frac{1}{\mu_{\alpha}^{\prime}} \cdot \frac{\Omega}{4 . \pi} \cdot C \cdot b_{\alpha} \cdot \sigma \cdot\left(1-e^{-\mu_{\alpha}^{\prime} \cdot d^{\prime}}\right) \prod_{i=0}^{n} e^{\left(-\mu_{\alpha}^{i} \cdot d^{i}\right)},
$$

where $\mu_{\alpha}^{\prime}$ and $\mu_{\alpha}^{i}[6]$, are the attenuation coefficient of the emitting layer and the layer $i$ placed ahead of the emitting one, respectively, $d^{\prime}$ and $d^{i}$ the $\mathrm{X}$-rays path in, the emitting layer and the layer $i$, which are a function of the detection angle $\theta . \epsilon$ is the detector efficiency, $b$ the line intensity faction [7], $C$ the concentration of the emitting element, $\Omega$ the detector solid angle, $\sigma$ the cross section of the considered X-ray emission, $N_{p}$ the number of protons crossing the target and $n$ the number of layers placed ahead of the emittiong one. In this equation, $\sigma$ is considered as a constant due to its low variation (when using high energy beams). The $\frac{K_{\alpha}}{K_{\beta}}$ ratio, $R$, is given by the equation

$$
R=\frac{N_{K_{\alpha}}}{N_{K_{\beta}}}=\frac{\mu_{\beta}}{\mu_{\alpha}} \cdot \frac{\epsilon_{\alpha}}{\epsilon_{\beta}} \cdot \frac{b_{\alpha}}{b_{\beta}} \cdot \frac{\left(1-e^{\left(-\mu_{\alpha}^{\prime} \cdot d^{\prime}\right)}\right)}{\left(1-e^{\left(-\mu_{\beta}^{\prime} \cdot d^{\prime}\right)}\right)} \cdot \prod_{i=0}^{n} \frac{e^{\left(-\mu_{\alpha}^{i} \cdot d^{i}\right)}}{e^{\left(-\mu_{\beta}^{i} \cdot d^{i}\right)}} .
$$



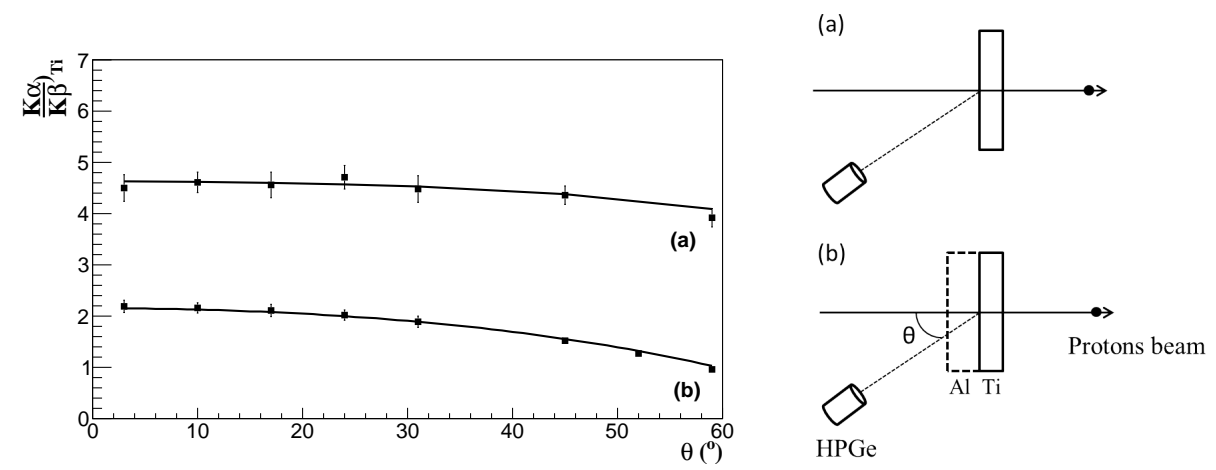

Figure 1: Evolution of $\frac{K_{\alpha}}{K_{\beta}}$ ratio as a function of the detection angle using 68 $\mathrm{MeV}$ proton beam: (a) for a single layer of $10 \mu \mathrm{m}$ of titanium, (b) for a layer of $50 \mu \mathrm{m}$ of aluminium placed ahead of the emitting one $(10 \mu \mathrm{m}$ of titanium). The schemas represent those two experimental configurations. Dots are the experimental data and the lines correspond to the values calculated using equation (2).

Figure 1 (a) shows both the experimental and the modelised variation of the $\frac{K_{\alpha}}{K_{\beta}}$ ratio in a single layer of titanium. The $\frac{K_{\alpha}}{K_{\beta}}$ ratio slighty varies in the layer itself meaning that it is barely sensitive to self attenuation. The variation of the self attenuation as a function of the detection angle is below the experimental errors, expected to be around $5 \%$. Thus we consider the ratio $\frac{K_{\alpha}}{K_{\beta}}$ as a constant. Figure 1 (b) shows the variation of the $\frac{K_{\alpha}}{K_{\beta}}$ ratio of the same layer of titanium with a aluminium layer placed ahead of the titanium. In this case, the ratio is dependent on a layer ahead of the emitting one. The ratio $\frac{K_{\alpha}}{K_{\beta}}$ contains the information about the amount of material in front of the considered layer and can be used to obtain the sequences and the thicknesses of a multi-layer target.

The relative variation of the $\frac{K_{\alpha}}{K_{\beta}}$ ratio as a function of the detection angle is given by

$$
\frac{\Delta R}{R}=\frac{R\left(\theta_{1}\right)-R\left(\theta_{2}\right)}{R\left(\theta_{1}\right)}=\frac{e^{\left(\frac{\Delta \mu \cdot d}{\cos \left(\theta_{1}\right)}\right)}-e^{\left(\frac{\Delta \mu \cdot d}{\cos \left(\theta_{2}\right)}\right)}}{e^{\left(\frac{\Delta \mu \cdot d}{\cos \left(\theta_{1}\right)}\right)}}
$$

where $\Delta \mu=\mu_{K_{\beta}}-\mu_{K_{\alpha}}$. With some algebra we can extract :

$$
\Delta \mu \cdot d=\frac{1}{\frac{1}{\cos \left(\theta_{2}\right)}-\frac{1}{\cos \left(\theta_{1}\right)}} \cdot \ln \left(\frac{1}{1-\frac{\Delta R}{R}}\right) .
$$


$\Delta \mu$.d contains the density, the chemical composition and the thickness of the effective layer between the emitting layer and the detector (the effective layer has the same effect on the X-rays attenuation than the sum of the real layers). Table 1 contains the values of $\Delta \mu \cdot d$ determined using equation 4 for the titanium layer in the configuration (b) of the figure 1 . We only use the combinations of $\theta$ inducing the larger variation of $\mathrm{R}$. Results shows that $\Delta \mu \cdot d$ is independent of the choice of $\theta_{1}$ and $\theta_{2}$. Errors on $\Delta \mu \cdot d$ are mainly due to the error on $\frac{\Delta R}{R}$ and so to the error on the number of detected X-rays.

Table 1: The values of $\Delta \mu \cdot d$ calculated using equation 4 for the titanium layer in the experimental configuration (b) in figure 1.

\begin{tabular}{ccc}
\hline$\theta_{1}\left(^{\circ}\right)$ & $\theta_{2}\left(^{\circ}\right)$ & $\Delta \mu \cdot d($ titanium $)$ \\
\hline 45 & 3 & $-0.886 \pm 0.058$ \\
45 & 10 & $-0.876 \pm 0.055$ \\
45 & 17 & $-0.891 \pm 0.054$ \\
45 & 24 & $-0.930 \pm 0.056$ \\
45 & 31 & $-0.916 \pm 0.056$ \\
\hline
\end{tabular}

This term is also function of the X-ray energy thus implying that equation (4) cannot be used in this form to determine the sequences. Lets take into account the energy dependency of the photoeletric mass attenuation coefficient [8].

$$
\frac{\mu}{\rho}=b \cdot \frac{1}{E^{\frac{7}{2}}} \cdot Z^{5} \cdot \frac{N_{A}}{A}
$$

where $E$ is the energy of the considered X-ray, $N_{A}$ is the Avogadro constant, $Z$ the atomic number, $b$ a constant and $A$ the mass number. Now we can calculate a new parameter $(\Delta \mu \cdot d)^{\prime}$ using equation 6 .

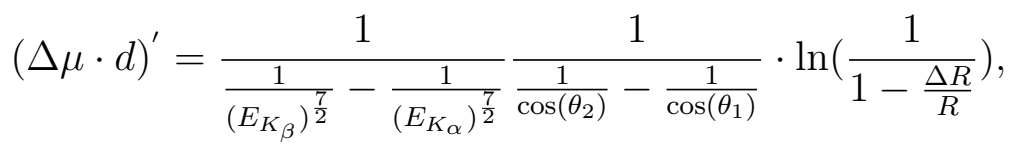

where $(\Delta \mu \cdot d)^{\prime}$ is also equal to $\rho \cdot d \cdot Z^{5} \cdot b \cdot \frac{N_{a}}{A} \cdot(\Delta \mu \cdot d)^{\prime}$ is a function of the properties of the effective layer between the detector and the considered layer. The smallest value of $(\Delta \mu \cdot d)^{\prime}$ represents the lowest quantity of matter crossed by the X-rays. The comparison between the $(\Delta \mu \cdot d)^{\prime}$ values of each detected element allows to determine the sequences. 
Using $\Delta \mu$.d given by equation (4) and equation (5), we can calculate the attenuation of $K_{\alpha}$ X-rays (or $K_{\beta}$ ) $M_{K_{\alpha}}$.

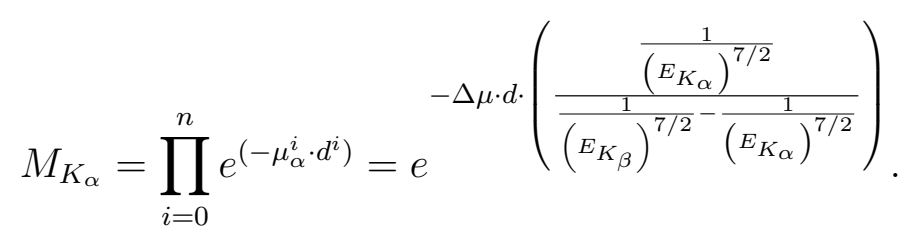

We can therefore correct the attenuation of the detected number of $K_{\alpha}$ X-rays, $N_{K_{\alpha}}$, by using equation (7). The thickness of each layer is given by the comparison between the equation 1 for the layers and for the standard samples (irridiated in the same condition).

$$
e_{\text {layer }}=-\frac{1}{\mu_{\alpha}^{\prime}} \cdot \ln \left(1-\frac{N_{K_{\alpha}}^{\text {layer }}}{N_{K_{\alpha}}^{\text {standard }} \cdot M_{K_{\alpha}}}\right) .
$$

The errors on the thicknesses are calculated using the propagation error formula and the associated error of $\Delta \mu \cdot d, N_{K_{\alpha}}^{\text {layer }}$ and $N_{K_{\alpha}}^{\text {standard }}$.

\section{Experimental set-up}

PIXE experiments were carried out at ARRONAX cyclotron [9] using 68 $\mathrm{MeV}$ protons. The experimental set-up used is described in more details in [5]. The X-rays are detected by a High Purity Germanium (HPGe) detector. The distance between the sample and the detector is $25 \mathrm{~cm}$. The sample holder can be tilted with respect to the incident beam in order to change the angle between the sample and the detector. We use a thin foil of copper (2 $\mu \mathrm{m})$ in order to monitor the number of particles crossing the target. This monitor foil is placed ahead of every target and is in contact with the samples.

\section{Investigated targets}

The analysis method described above is used on both ideal targets, composed of pure material foils with no repetition of the same elements, similar to the multi-layers targets studied in [5] and on more complex cases, where some layers are hidden. The targets used in this work are listed in Table 1.

Table 2: Composition of the investigated targets. The position gives the location of the layer inside the target. Position 1 is closest to the surface and 3 (or 4) is the last position. 


\begin{tabular}{cc|cc}
\multicolumn{2}{c|}{ Target 1 } & \multicolumn{2}{c}{ Target 2 } \\
\hline \hline Element & Position & Element & Position \\
\hline Titanium & 1 & Aluminium & 1 \\
Silver & 2 & Titanium & 2 \\
Gold & 3 & Aluminium & 3 \\
- & - & Silver & 4 \\
\hline
\end{tabular}

In this particular set-up aluminium is used to simulate hidden layers due to the high attenuation of low energy X-rays emitted by aluminium.

Standard targets for each detected element have also been irradiated in the same experimental condition.

\section{Results and Discussion}

Figure 2 shows spectra of the studied targets. In both targets, X-rays from the monitor foil of copper are detected. We can notice that the X-rays from aluminium $(1.486 \mathrm{keV})$ are not visible in the spectrum of the second target.
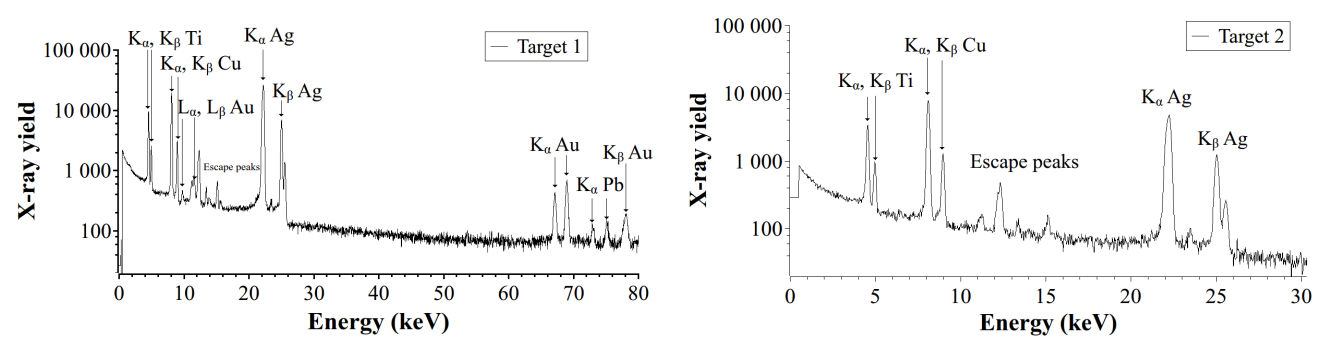

Figure 2: Spectra of the investigated targets obtained using $68 \mathrm{MeV}$ proton beam at the detection angle of $3^{\circ}$. First spectrum: ideal sample consisting of thick silver and gold layers. Second spectrum: sample consisting of two thick aluminium, titanium and silver layer. The X-ray from copper visible in those spectra are due to the presence of the monitor foil.

Using equations (6) and (8), we have determined the $(\Delta \mu \cdot d)^{\prime}$ value and the thicknesses of the foils for each target (Table 2). The experimental error on the $(\Delta \mu \cdot d)^{\prime}$ value and the calculated thickness are only due to the statistical error on the detected K-lines number and the detector efficiencies. The calculated thicknesses and the sequences for the first target are in a good agreement with the calibrated thicknesses and the positions of each layers. 
The value of $(\Delta \mu \cdot d)^{\prime}$ for titanium is not as close to zero as it should be (because it's the first layer). This is due to the monitor foil.

For the more complex target, the calculated thicknesses of the two elements detected are in a good agreement with the real see Table 3) even though the two aluminium layers aren't detected. The values of $(\Delta \mu \cdot d)^{\prime}$ for the titanium and the silver are far from the value of the simple target, which means there is one or more layer in front of the titanium and between the titanium and the silver. The high values for $(\Delta \mu \cdot d)^{\prime}$ reveal the presence of some hidden layers in the sample.

Table 3: Results of the multi-layers analysis using detection angles of $3^{\circ}$ and $45^{\circ}$

Target 1

\begin{tabular}{cccc}
\hline Element & $(\Delta \mu \cdot d)^{\prime}$ & Calculated thickness $(\mu \mathrm{m})$ & Calibrated thickness $(\mu \mathrm{m})$ \\
\hline Titanium & $92 \pm 6$ & $10.40 \pm 0.62$ & $10.03 \pm 0.02$ \\
Silver & $860 \pm 40$ & $25.82 \pm 1.29$ & $24.87 \pm 0.04$ \\
Gold & $12200 \pm 600$ & $9.11 \pm 0.59$ & $10.74 \pm 0.02$ \\
& & & \\
\hline Target 2 & & & $10.06 \pm 0.02$ \\
\hline Titanium & $664 \pm 62$ & $9.25 \pm 0.93$ & $10.32 \pm 0.02$ \\
Silver & $4168 \pm 348$ & $10.33 \pm 0.21$ & \\
\hline
\end{tabular}

\section{Conclusions}

High energy PIXE provides high indepth analysis and a simpler analysis due to the range of high energy beam, the slow variation of the ionization cross section and the low energy loss of $68 \mathrm{MeV}$ protons beam. The self attenuation in the emitting layer has little impact on the variation of $\frac{K_{\alpha}}{K_{\beta}}$ as a function of the detection angle but this ratio is sensitive to the layers located above and contains information about those layers. Those properties allowed us to use this ratio to determine the thicknesses and the sequences of the layers in a target which contained undetected layers at the surface and/or in the bulk. Furthermore, we dont't have to make any hypothesis on the composition of the undetected layers in order to perform the analysis. 


\section{Acknowledgments}

The ARRONAX cyclotron is a project promoted by the Regional Council of Pays de la Loire financed by local authorities, the French government and the European Union. This work has been, in part, supported by a grant from the French National Agency for Research called Investissements dAvenir, Equipex Arronax-Plus no ANR-11-EQPX-0004 and Labex no ANR11-EQPX-0018-01.

\section{Reference}

[1] A. Denker, K. Maier, Investigation of objects d'art by PIXE with 68 mev protons, Nuclear Instruments and Methods in Physics Research B 161-163 (2000) 704-708.

[2] A. Denker, M. Blaich, PIXE analysis of middle age objects using 68 mev protons, Nuclear Instruments and Methods in Physics Research B 189 (2002) 315-319.

[3] T. Dupuis, Preliminary experiments : High-energy alpha PIXE in archaeometry, Nuclear Instruments and Methods in Physics Research B 268 (2010) 1911-1915.

[4] A. Denker, J. Optiz-Coutureau, Paintings- high-energy protons detect pigments and paint-layers, Nuclear Instruments and Methods in Physics Research B 213 (2004) 677-682.

[5] D. Ragheb, al, Development of a PIXE method at high energy with the arronax cyclotron, Journal of Radioanalytical and Nuclear Chemistry 301 (2014) 895-901.

[6] National Institute of Standards and Technology (Jun. 2016).

URL http://physics.nist.gov/PhysRefData/Xcom/html/xcom1. html

[7] J. Scofield, Exchange corrections of k x-ray emission rates, Physical Review A 9 (1974) 1041-1049.

[8] W. Heitler, The quantum theory of radiation, Oxford Univ Press 207-208. 
[9] F. Haddad, al, Arronax a high-energy and high-intensity cyclotron for nuclear medicine, European Journal of Nuclear Medicine and Molecular Imaging 35 (2008) 1377-1387. 